\title{
A fast boundary protection for an AC transmission line connected to an LCC- HVDC inverter station
}

\author{
Zhen Liu', Houlei Gao ${ }^{1 *}$, Sibei Luo ${ }^{1}$, Le Zhao ${ }^{2}$ and Yuyao Feng ${ }^{2}$
}

\begin{abstract}
For AC transmission lines connected to an LCC-HVDC inverter station, commutation failure can lead to the wrong operation of traditional protection. To solve the problem, this paper proposes a fast protection scheme using transient information from one end of the AC line. The boundary frequency characteristics of the AC line connected to LCC-HVDC inverter are analyzed first. This reveals the existence of significant attenuation on both high frequency signals and some specific frequency signals. Based on the boundary characteristics, a novel boundary protection principle utilizing a backward traveling wave is then proposed. A PSCAD/EMTDC simulation model of a $\pm 800 \mathrm{kV}$ LCC-HVDC and $500 \mathrm{kV}$ AC transmission system is established, and different fault cases are simulated. The simulation results prove that the novel protection principle is immune to commutation failure, fault resistance and fault type.
\end{abstract}

Keywords: LCC-HVDC, Commutation failure, Transient protection, AC transmission line, Inverter station, Boundary characteristics

\section{Introduction}

An HVDC transmission system has many advantages over an AC transmission system such as long transmission distance, high efficiency and large transmission capacity [1-6]. However, as an LCC-HVDC inverter station is severely affected by an AC transmission line (ACTL) fault, it is important that an ACTL fault is cleared quickly and reliably. Otherwise, it could lead to commutation failure or even an HVDC system blocking [7-12]. Commutation failure and complex electrical dynamics during the recovery process result in the backside system impedance exhibiting nonlinear time-varying characteristics, whereas the traditional AC system fault analysis theory is based on linear systems. Thus, the adaptability of conventional power frequency protection (PFP) in LCC-HVDC connected AC/DC hybrid systems has

\footnotetext{
* Correspondence: houleig@sdu.edu.cn

'Key Laboratory of Power System Intelligent Dispatch and Control of Ministry of Education, Shandong University, No. 17923, Jingshi Road, Jinan 250061,

China

Full list of author information is available at the end of the article
}

problems, which have been extensively and deeply studied [13-18].

When a commutation failure occurs due to an AC system fault, the amplitude of the equivalent power frequency current decreases and the fluctuation range of the phase angle is large. This may cause current differential protection to fail [14]. In addition, the directional pilot protection principle cannot adapt to an ACTL connected to an inverter station [15]. Since the positive and negative sequence impedances are not equal for an ACTL connected to an inverter station, it may lead to mis-operation of the directional element using sequence components [17]. The DC part exhibits a large shortcircuit impedance when an AC line fault occurs, and this can cause distance protection to reduce or lose its protection range [16], while the main electromagnetic transient process caused by commutation failure influences AC line distance protection [18].

However, theoretical analysis and on-site recording indicate that the time interval from fault to first commutation failure is generally more than $3 \mathrm{~ms}[19,20]$. 
Therefore, if protection can activate a tripping order for an ACTL fault within $3 \mathrm{~ms}$ of the fault, the consequent commutation failure will not affect the correct action of the protection. However, none of the power frequencybased protection can meet such a requirement, and it is only possible using transient-based protection.

Transient-based protection directly utilizing fault transient information to quickly remove a fault has been studied over the past four decades [21-27]. It can be divided into unit and non-unit transient-based protection from the perspective of space utilization information. Compared with unit transient protection, non-unit transient protection only uses locally measured transient fault information and can accomplish ultra-high-speed operation without communication delay. Traveling wave distance protection [23] and boundary protection are both non-unit protection. Boundary protection can distinguish internal and external faults from the fault characteristic differences caused by the change of surge impedance at the line boundary.

The line boundary in a primary system and boundary characteristics determine the performance of boundary protection. The earliest line boundary is the conventional line trap and specially designed stack tuner aimed at capturing a high-frequency voltage signal in EHV transmission systems [28, 29]. However, adjusting the line trap and installing a stack tuner are difficult in practice. The busbar stray capacitance is regarded as the line boundary in [30]. This has severe attenuation on high-frequency transient current. Some researchers consider both line trap and busbar stray capacitance as line boundary [31]. Since fiber optic communication is now widely used, line traps are rarely used and the scope of their application is limited. To improve the performance of boundary protection, the line boundary and corresponding characteristics in UHVAC transmission systems have been analyzed comprehensively based on real projects. Busbar stray capacitance, the shunt reactor, series capacitance and combined line boundary are used as the line boundary to distinguish internal and external faults considering fault characteristic differences, while a more precise setting method is proposed [1].

Compared with that for the AC transmission line, the development of boundary protection for the DC transmission line has made great progress. In the primary HVDC transmission system, DC filters and smoothing reactors at each line terminal constitute the HVDC line boundary. This line boundary imposes a significant attenuating or smoothing effect on transient signals, and makes significant characteristic differences between internal and external faults [32-36]. However, study on composition and characteristics of an ACTL connected to an inverter station is still limited.
The extent of full usage of the fault characteristic differences caused by line boundary also determines the performance of boundary protection. Digital filters [29, 30], wavelet transform, neural network and mathematical morphology are applied in boundary protection algorithms [37-40], but they still rely on the line boundary composed of busbar stray capacitance [37-40].

To develop a novel protection not affected by commutation failure, a boundary protection (BP) principle utilizing a backward traveling wave is proposed because of the boundary specificity of an ACTL connected to an inverter station. The proposed method can respond to faults quickly (in under $3 \mathrm{~ms}$ ) and is immune to commutation failure. The boundary composition and characteristics of the AC line connected to an LCC-HVDC inverter are analyzed first and based on the boundary characteristics, the novel boundary protection principle using a backward traveling wave is then proposed. A synchronous squeeze wavelet transform-based algorithm is established to extract the energy of a narrow band signal. Finally, the effectiveness of the proposed protection is validated by simulation tests of the $\pm 800 \mathrm{kV}$ HVDC system in China.

This rest of the paper is organized as follows. Boundary characteristic analysis is investigated in Section 2 and the BP algorithm using a backward traveling wave is described in Section 3. The simulation model of the \pm $800 \mathrm{kV}$ LCC-HVDC and $500 \mathrm{kV}$ AC transmission system is established in Section 4, and extensive simulations are carried out to verify the effectiveness of the proposed protection. Finally, conclusions are drawn in Section 5.

\section{Boundary characteristics analysis}

\subsection{Boundary composition}

The line boundary is the discontinuous point of the surge impedance of a transmission line, and is usually located at both ends of a protected line. Based on the parameters of the Tian-Zhong $\pm 800 \mathrm{kV} / 8000 \mathrm{MW}$ HVDC system in China, a typical DC-AC hybrid system is shown in Fig. 1, where Cs represents the busbar stray capacitance. As shown, in order to compensate for the reactive power absorption of the LCC converter and restrain the generated AC harmonics, AC filters and a reactive power compensation device (RPCD) are installed on the busbar connected to the AC transmission lines. DC filters and a smoothing reactor (SR) are installed at both ends of the DC transmission line to retrain DC harmonics. These reactive power elements form both the AC boundary and the DC boundary of the inverter station as shown in Fig. 1.

\subsection{Frequency characteristics of AC boundary of inverter station}

As can be seen in Fig. 1, the AC boundary consists of the AC filters, RPCD and EHV busbar capacitance. The 


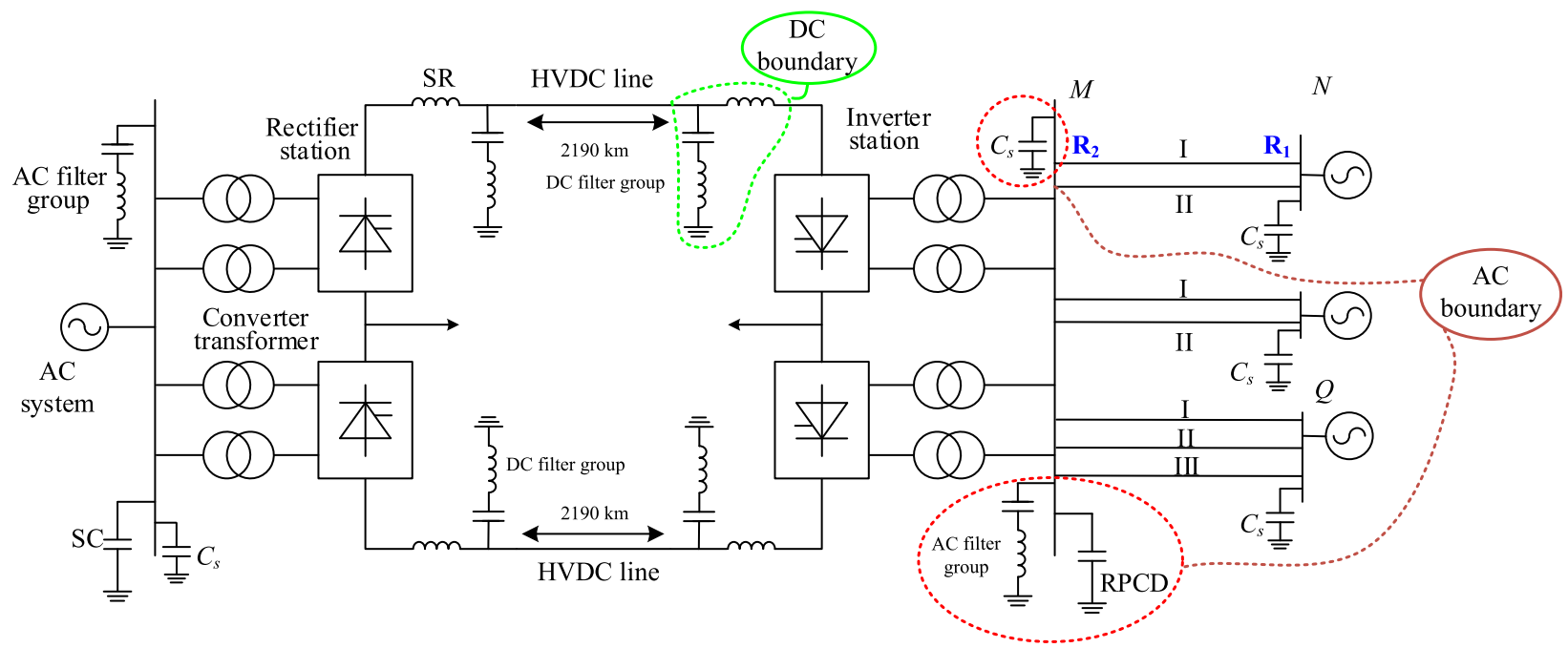

Fig. 1 DC-AC hybrid system

parameters and structure of the AC filters and RPCD can be obtained from [41]. The configuration of the inverter station $\mathrm{AC}$ filters and shunt capacitors is shown in Table 1.

The reactive power compensation device is a high voltage shunt capacitor (SC), which generally consists of a high voltage capacitor and a small damping reactance connected in series. The purpose of the damping reactance is to limit inrush current when the capacitor is switched in. As indicated in some studies, the inrush current generated when switching in a single group of capacitors is not severe, and thus a single shunt capacitor can be switched directly without the use of the series reactance. However, when an existing capacitor group is already connected and another group is switched in, the inrush current can be very large and the small damping reactance is required. Considering the economical aspect, there is only one set of shunt capacitors not having the small damping reactance in the whole station [42]. The small damping reactance ranges from 1 to $5 \mathrm{mH}$, with a typical value of around $2 \mathrm{mH}$. Therefore, the high

Table 1 Parameters of the AC filters and shunt capacitors

\begin{tabular}{lllll}
\hline Elements & \multicolumn{3}{l}{ Type of AC filters } & $\begin{array}{l}\text { Capacity of filter } \\
\text { group }\end{array}$ \\
\cline { 2 - 4 } & HP12/24 & HP3 & SC & \begin{tabular}{l} 
3-Phase /Mvar \\
\hline$C_{1} / \mathrm{mF}$
\end{tabular} \\
$\mathrm{L}_{1} / \mathrm{mH}$ & 7.992 & 3.003 & 3.01 & 1 st, 2nd and 3rd group: \\
$\mathrm{C}_{2} / \mathrm{mF}$ & 10.194 & 24.021 & & \\
$\mathrm{~L}_{2} / \mathrm{mH}$ & 4.418 & & & \\
$\mathrm{R}_{1} / \Omega$ & 1000 & 1060 & & 4th group: \\
Tuned frequency/Hz & $600 / 1200$ & 150 & & 1040 \\
Q3p, at 515 kV/Mvar & 260 & 260 & 260 & Total: \\
Groups of filters & 8 & 2 & 9 & 4940 \\
\hline
\end{tabular}

voltage shunt capacitors are divided into two cases: a shunt capacitor with a small damping reactance (SC) and shunt capacitor without the damping small reactance (SCO).

The detailed filter arrangements for the 4 large filter groups at the inverter station are as follows:

- Groups 1 and 2: each contains two HP12/24 filters, one HP3 filter and 2 SC.

- Group 3: two HP12/24 filters and 3 SC.

- Group 4: two HP12/24 filters and 2 SC, of which one $\mathrm{SC}$ has no small damping reactance $(\mathrm{SCO})$.

The AC filters and RPDC are all connected in parallel, which is the same as the ground stray capacitance for the AC substation [1]. The case of the line boundary is shown in Fig. 2a and the lumped parameter equivalent circuit in the Laplace domain is depicted in Fig. 2b. Because the surge impedance is discontinuous at the line boundary of a transmission line whose surge impedance is marked as $Z_{1}$ in Fig. 2, the incident traveling wave $u_{1 b}$ generated by a fault is reflected to form the reflected wave $u_{1 f}$. It is then refracted to form the refracted wave $u_{2 f}$, which enters the other transmission line whose surge impedance is $Z_{2}$. Therefore, these devices connected in parallel have the generic boundary transfer characteristic expression:

$$
H_{\text {para }}(\omega)=\frac{2\left(Z_{2} / / Z_{\text {para }}\right)}{Z_{1}+Z_{2} / / Z_{\text {para }}}
$$

where sign // represents parallel relation; $H(\omega)$ denotes the transfer characteristic of the line boundary, i.e. the refractive coefficient, which is related to the parameters 


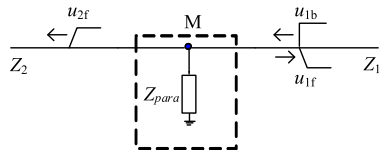

(a)

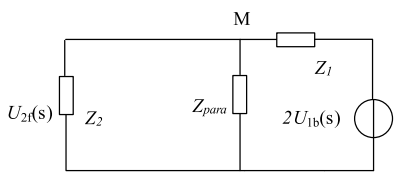

(b)

Fig. 2 Refraction and reflection of traveling wave at the line boundary consisting of parallel impedance. a Line boundary. b Lumped parameter equivalent circuit by the Peterson principle

of the line boundary and the line surge impedances $Z_{1}$ and $Z_{2}$.

Assuming $Z_{1}=Z_{2}=250 \Omega$, then based on the Peterson principle and the system parameters, the AC boundary characteristics of the inverter station when there is an individual AC filter, the SC and filter group are considered separately can be respectively obtained, as shown in Fig. 3 and Fig. 4.

The amplitude-frequency characteristics of the AC boundary are summarized as follows:

a) The reactive power compensation shunt capacitor without small damping reactance (SCO) is equivalent to directly connecting a large capacitor to the ground, which is tens to hundreds of times the stray capacitance of the busbar to ground in an $\mathrm{AC}$ system, and the line boundary formed has

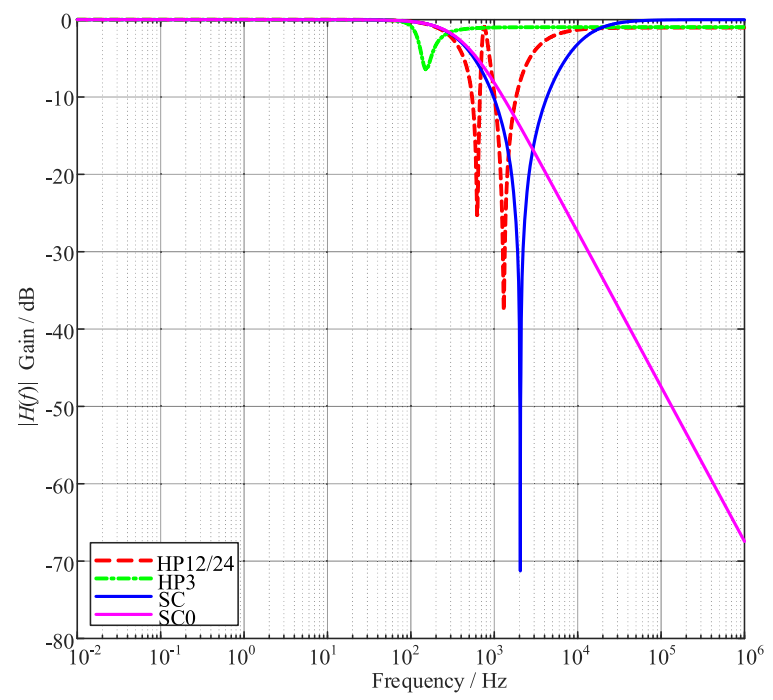

Fig. 3 Amplitude-frequency characteristic of the line boundary consisting of AC filter or SC

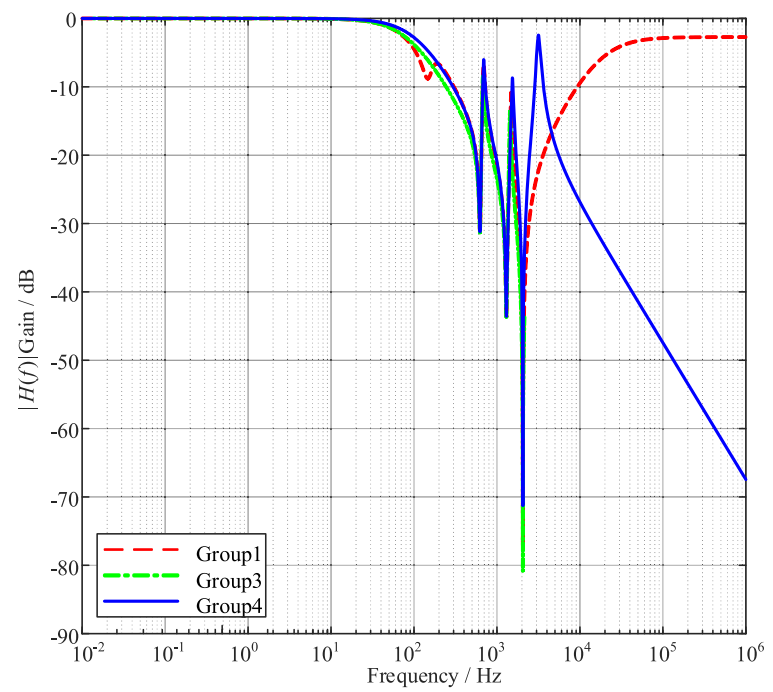

Fig. 4 Amplitude-frequency characteristic of the line boundary of each filter group

significant attenuation on high frequency signals above $10 \mathrm{kHz}$.

b) The line boundary formed by the shunt capacitor with small reactance (SC) only has attenuation on a narrow band signal centered on the resonance frequency generated by the small reactance and capacitor, while it has no obvious attenuation on other frequency components. This resonance frequency and the narrow band signal are called the specific frequency and specific frequency component, respectively.

c) The line boundary formed by the AC filter (HP12/ 24) has attenuation only on a narrow band signal centered on the tuned frequency, while it has no obvious attenuation on other frequency components. Similarly, this tuned frequency is the specific frequency while the narrow band signal is the specific frequency component. The line boundary formed by the low-order harmonic filter (HP3) has limited attenuation on the narrow band signal centered at the tuned frequency of $150 \mathrm{~Hz}$.

d) The boundary characteristics of each of the 3 largegroup filters comprehensively reflect and strengthen the boundary characteristics when considered alone and have significant attenuation on a specific frequency signal and high frequency signals.

Based on the above analysis, it can be concluded that the AC boundary of an LCC-HVDC inverter station has significant attenuation both on high frequency signals and specific frequency signals. These will cause different fault characteristics between internal and external faults. 


\subsection{Frequency characteristics of DC boundary of the inverter station}

DC filters and the smoothing reactor form the DC boundary of the inverter station. The parameters and structure of the DC filters and SR can be obtained from [41]. The DC filters are all connected in parallel, which is the same as the AC filters and RPDC, while the smoothing reactor is connected in series. The configuration of the inverter station DC filters is shown in Table 2. The case of the line boundary is shown in Fig. 5a and the lumped parameter equivalent circuit in the Laplace domain is depicted in Fig. 5 (b). Therefore, these devices connected in series have the generic boundary transfer characteristic expression:

$$
H(\omega)=\frac{2 Z_{2}}{Z_{1}+Z_{\mathrm{SR}}+Z_{2}}
$$

The DC boundary characteristics of the inverter station are studied in the following ways:

- DC filter group only (HP12/24 and HP2/39);

- SR only, with the inductances commonly used 75, 150, 225, 300 and $400 \mathrm{mH}$;

- Combination of DC filters and SR with different inductance values.

The amplitude-frequency characteristics of the above line boundaries are shown in Fig. 6 (a), (b), and (c).

The amplitude-frequency characteristics of the DC boundary are summarized as follows:

a) The boundary formed by the DC filters (HP12/24 and HP3/39) has no attenuation effect on high frequency signals, whereas it has significant attenuation on a narrow band signal centered on the tuned frequency (called as specific frequency). The line boundary formed by the DC filter (HP3/ 39) has limited attenuation on the narrow band signal centered at the tuned frequency of $100 \mathrm{~Hz}$.

b) The line boundary formed by SR has a significant attenuation effect on high frequency signals above

Table 2 Parameters of DC filter

\begin{tabular}{lll}
\hline Elements & HP12/24 & HP3 \\
\hline $\mathrm{C}_{1} / \mathrm{mF}$ & 0.35 & 0.8 \\
$\mathrm{~L}_{1} / \mathrm{mH}$ & 89.35 & 11.99 \\
$\mathrm{C}_{2} / \mathrm{mF}$ & 0.81 & 1.825 \\
$\mathrm{~L}_{2} / \mathrm{mH}$ & 48.86 & 964 \\
$\mathrm{R}_{1} / \Omega$ & 10,000 & 5700 \\
Tuned frequency/Hz & $600 / 1200$ & $100 / 1950$ \\
\hline
\end{tabular}

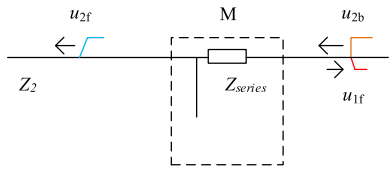

(a)

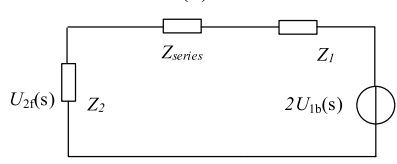

(b)

Fig. 5 Refraction and reflection of traveling wave at the line boundary consisting of series impedance. a Line boundary. $\mathbf{b}$ Lumped parameter equivalent circuit by the

Peterson principle

$10 \mathrm{kHz}$. A larger SR inductance value leads to higher attenuation.

c) The combination of the DC filters and SR comprehensively reflects and strengthens the boundary characteristics. This makes the attenuation of specific frequency components and high frequency components more severe.

Based on the above analysis, it can be concluded that the DC boundary of an LCC-HVDC inverter station has significant attenuation on high frequency signals and specific signals. The characteristics will make significant differences between high frequency or specific frequency components of the internal fault and the external fault. These can be used to form the boundary protection.

The AC boundary and DC boundary of the inverter station together form the boundary of the ACTL connected to the LCC-HVDC inverter station. By considering the frequency characteristics indicating that they have significant attenuation on both the specific frequency components and high frequency components, a new boundary protection principle can be formed.

\section{Principle and algorithm of boundary protection 3.1 Protection principle}

Assuming the current reference direction at the relaying point is from the busbar to the protected line and observing the relaying point, the forward traveling wave $\Delta u_{f}$ and the backward traveling wave $\Delta u_{b}$ are:

$$
\begin{aligned}
& \Delta u_{\mathrm{f}}=\frac{1}{2}(\Delta u+Z \Delta i) \\
& \Delta u_{\mathrm{b}}=\frac{1}{2}(\Delta u-Z \Delta i)
\end{aligned}
$$

where $\Delta u$ and $\Delta i$ are the fault components of voltage and current measured at the relaying point, respectively. $Z$ denotes the surge impedance of the protected line. 


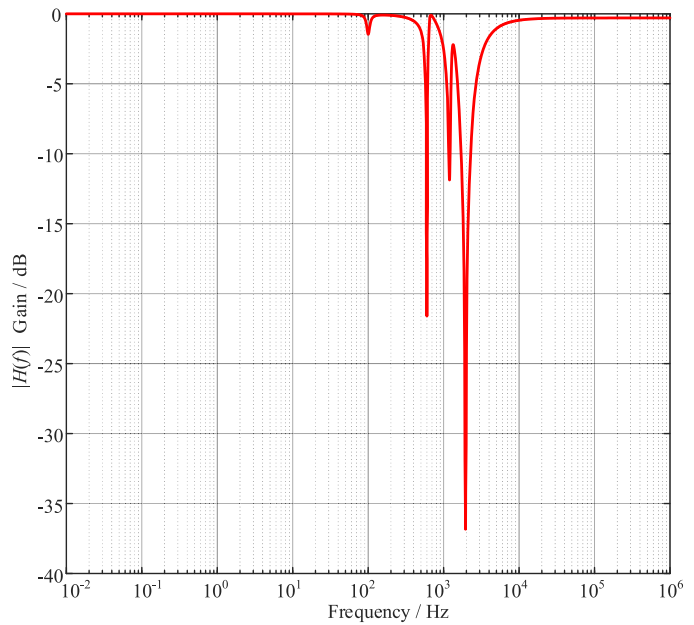

(a)

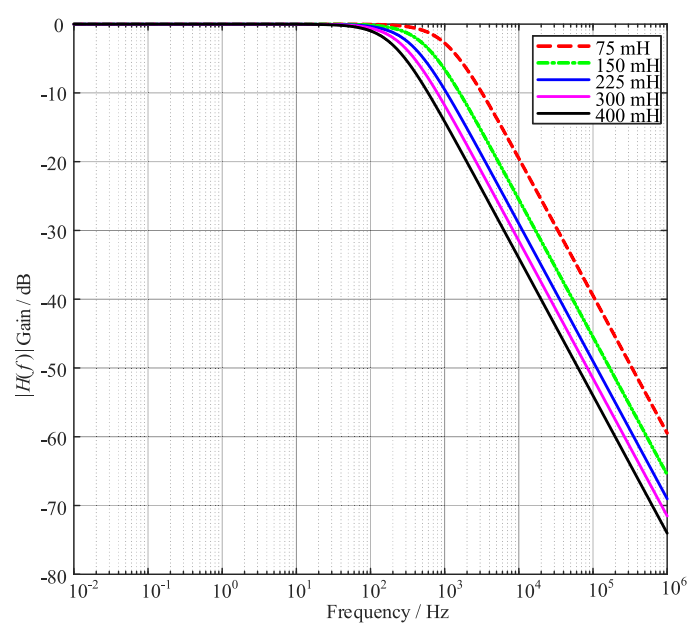

(b)

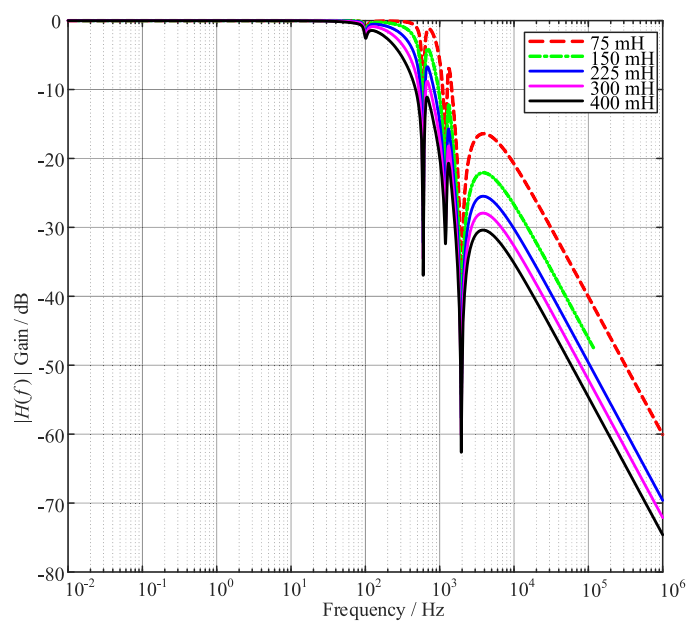

(c)

Fig. 6 Amplitude-frequency characteristic of DC boundary of the inverter station
Assuming boundary protection is located at the line terminal $\mathrm{M}$ of the protected line $\mathrm{MN}$ as shown in Fig. 1, in the case of an internal fault at $f_{1}$, the Bewley lattice diagram of the fault generated traveling waves is shown in Fig. 7. $B_{1}$ and $F_{1}$ are the initial backward and forward traveling waves, respectively. The subsequent backward and forward traveling waves mainly include the following:

- $\mathrm{B}_{2}$ and $\mathrm{F}_{2}$ stemming from the opposite terminal boundary $\mathrm{N}$;

- $\mathrm{B}_{3}$ and $\mathrm{F}_{3}$ stemming from the fault point $\mathrm{f}_{1}$;

- $\mathrm{B}_{4}$ and $\mathrm{F}_{5}$ stemming from the forward external line boundary P;

- $\mathrm{F}_{4}$ from the backward external line boundary $\mathrm{Q}$.

In Fig. 7, the initial backward traveling wave $B_{1}$ arrives at the relaying point $\mathrm{M}$, and then boundary protection detects a fault occurrence at time $t_{0}$. Before $\mathrm{B}_{4}$ reaches the relaying point, i.e., within the time interval $\left[t_{0}, t_{0}+\right.$ $\Delta t$ ), all the backward traveling waves detected by boundary protection $\left(B_{1}, B_{2}\right.$ and $\left.B_{3}\right)$ do not pass through the line boundary. Thus, amplitudes of the high frequency and specific frequency components in the backward traveling waves will not be attenuated by the line boundary. When a fault occurs at the end of the protected line $\mathrm{MN}, \Delta t$ takes the minimum value as $\Delta t_{\min }=2 l_{N P} / c$, where $l_{N P}$ denotes the length of line NP, and $\mathrm{c}$ is the propagation velocity of the traveling wave.

In the case of a forward external fault at $f_{2}$, the Bewley lattice diagram of the fault generated traveling waves is depicted in Fig. 8. $B_{1}$ and $F_{1}$ are the initial backward and forward traveling waves, respectively. The subsequent backward and forward traveling waves mainly include the following:

- $\mathrm{B}_{2}$ and $\mathrm{F}_{2}$ stemming from the fault point $\mathrm{f}_{2}$;

- $\mathrm{B}_{3}$ and $\mathrm{F}_{3}$ stemming from the opposite terminal boundary $\mathrm{N}$;

- $\mathrm{B}_{4}$ and $\mathrm{F}_{4}$ from the forward external line boundary $\mathrm{P}$;

- $\mathrm{F}_{5}$ from the backward external line boundary $\mathrm{Q}$.

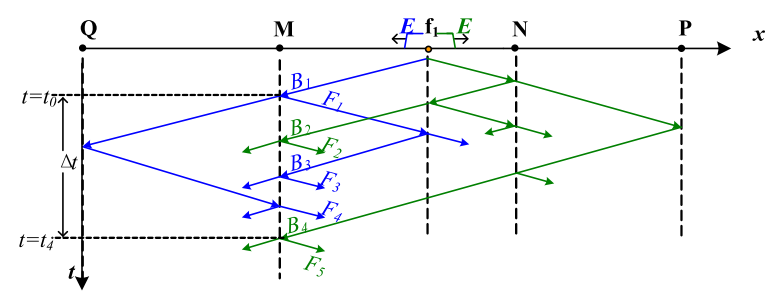

Fig. 7 Bewley lattice diagram under a forward internal fault 
Boundary protection detects the fault at time $t_{0}$. Before $\mathrm{B}_{3}$ arrives at the relaying point, i.e., within the time interval $\left[t_{0}, t_{0}+2 l_{M N} / c\right)$, where $l_{M N}$ denotes the length of line $\mathrm{MN}$, all the backward traveling waves detected by boundary protection $\left(B_{1}, B_{2}\right.$, when the line NP is short, $\mathrm{B}_{4}$ may also be included) do pass through the line boundary $\mathrm{N}$. Therefore, amplitudes of the high frequency and specific frequency components in the backward traveling waves will be attenuated by the line boundary significantly.

In the case of a backward external fault at $f_{3}$, the Bewley lattice diagram of the fault generated traveling waves is shown in Fig. 9. At the instant $t_{0}$, the initial forward traveling wave $F_{1}$ reaches the relaying point $M$, and boundary protection detects a fault occurrence at time $t_{0}$. Before $B_{1}$ (stemming from the opposite terminal boundary $\mathrm{N}$ ) arrives at the relaying point, i.e., within the time interval $\left[t_{0}, t_{0}+2 l_{M N} / c\right)$, the backward traveling wave does not appear. Thus, the backward traveling wave detected by boundary protection is zero and theoretically, $E_{\mathrm{f}} / E_{\mathrm{b}}$ becomes infinite. However, for a forward fault, the backward wave detected by boundary protection is the initial traveling wave and the subsequent backward and forward traveling waves meet the law of reflection, so within the time interval $\left[t_{0}, t_{0}+2 l_{M N} / c\right)$, the backward and forward traveling waves can be expressed as:

$$
\Delta u_{f}=k_{f} \Delta u_{b}
$$

where $k_{f}$ is the reflection coefficient of the bus.

Therefore, it can be concluded that within the time interval $\left[t_{0}, t_{0}+2 l_{M N} / c\right)$, the time-domain energy ratio of the forward and the backward traveling waves $E_{\mathrm{f}} / E_{\mathrm{b}}$ is less than 1 because $\left|k_{f}\right| \leq 1$.

From the above analysis, there exist significant differences in the backward traveling waves between internal and external faults. Thus, a novel principle of boundary protection is proposed to effectively utilize such differences.

After detecting an initial traveling wave generated by a fault, in the period of $\Delta t$, the time-domain energy ratio of the forward and backward traveling waves $E_{\mathrm{f}} / E_{\mathrm{b}}$, the energies of the high frequency and specific frequency components of the backward traveling waves $E_{h}$ and $E_{p}$,

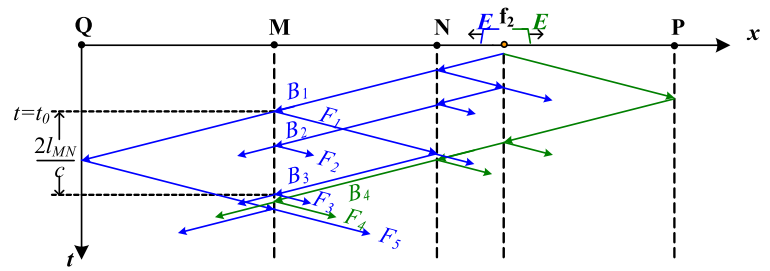

Fig. 8 Bewley lattice diagram under a forward external fault

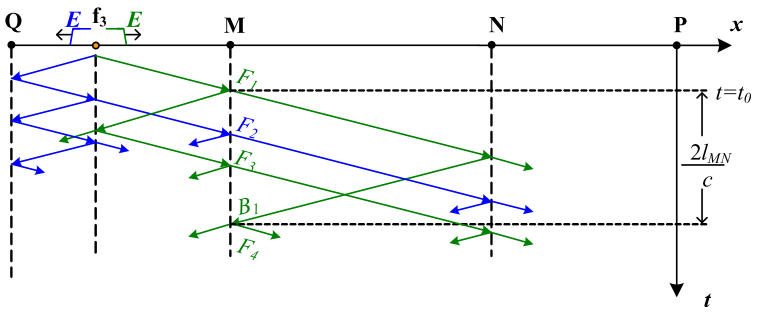

Fig. 9 Bewley lattice diagram under a forward external fault

are calculated respectively. If $E_{\mathrm{f}} / E_{\mathrm{b}}>\varepsilon_{0}$, the fault is identified as a backward external fault, otherwise it is a forward fault and the product of the high frequency energy and specific frequency energy $\left(E_{h} E_{\mathrm{r}}\right)$ is calculated. If $E_{h} E_{r}>\varepsilon_{1}$, the fault is determined to be internal, otherwise it is a forward external fault.

The protection principles can be further described as follows:

(1) The threshold $\varepsilon_{0}$ is used to distinguish the fault direction. In the case of backward faults, within the time interval $\Delta \mathrm{t}$, the backward traveling wave is theoretically zero. Thus, considering sensitivity and reliability, the threshold value $\varepsilon_{0}$ is 2 in this paper. To ensure that the fault direction characteristic is strictly established, $\Delta \mathrm{t}<2 l_{s h} / c$, where $l_{s h}$ is the length of the shortest line among all the lines connected to the same busbar.

(2) Transient current is a high frequency signal, which is easily influenced by high frequency noise. A mathematical morphology filter (MMF) with a flat structure element is applied to remove high frequency noise and eliminates the possibility of incorrect operation caused by the sharp part of the waveform.

(3) The length of the AC transmission line connected to the LCC-HVDC inverter station is short so that the post-fault time interval $\Delta t$ is very short. The shorter time interval $\Delta t$ makes the protection operation faster. However, in order to make better use of the characteristic differences of the backward traveling waves between internal and external faults, besides the initial backward traveling wave $B_{1}$, the subsequent backward traveling waves $\left(B_{2}\right.$ and/or $B_{3}$ ) under internal faults should also be included in $\Delta \mathrm{t}$, so $\Delta \mathrm{t}>l_{M N} / c$. Moreover, if $\Delta t$ is too short, the extraction for the high and specific frequency components will be difficult. Considering the protection operation speed and reliability, $\Delta \mathrm{t}$ should be set as $l_{M N} / c<\Delta t<\min \left\{2 l_{N P} / c, 2 l_{M N} / c\right\}$. For the protection line connected to the LCC-HVDC inverter station, $\Delta t=0.42 \mathrm{~ms}\left(l_{M N}=62.5 \mathrm{~km}\right)$ and it can be used to extract the energy. However, $\Delta t$ can be extended due to the attenuation on the magnitude of the 
traveling wave propagating on the transmission line. It has no effect on the accuracy of the boundary protection and can ensure reliability.

(4) The boundary elements of $R_{2}$ depend on the characteristics of the line boundary N. For ultrahigh voltage $\mathrm{AC}$ lines, the line boundary $\mathrm{N}$ consists of only the busbar ground stray capacitance which has significant attenuation on high frequency components [1]. Thus, the time-domain energy of the high frequency components can be used to identify the fault.

(5) Clarke phase-modal transformation is applied in this paper to obtain the $\alpha, \beta$ and 0 modal voltages and currents. Boundary protection uses the aerialmode components due to the severe attenuation of the zero-mode component. Comparing the absolute magnitudes of the $\alpha$ mode and $\beta$ mode, the larger is selected as the significant fault mode to cover all ten types of fault and form the backward traveling wave in the above boundary protection principle.

\subsection{Synchronous squeeze wavelet transform-based algorithm}

A synchronous squeeze wavelet transform (SST) transforms the time-scale $(a, b)$ plane onto the time-frequency $\left(\omega_{l}, b\right)$ planes to make $W_{x}(a, b)$ limited to the frequency range with the center frequency of $\omega_{l}$, by compressing the wavelet transform coefficients $W_{x}(a, b)$. In the frequency range where the center frequency is $\omega_{l}$, the timefrequency energy is concentrated near the center frequency and there is no cross term in each frequency curve, thereby effectively reducing band aliasing.

SST can obtain the band energy of the signal over a certain period $\left(\left[t_{1}, t_{2}\right]\right)$ in a certain frequency interval $\left(\left[f_{1}, f_{2}\right]\right)$, as shown in (6). Compared with DWT, SST divides the frequency space more precisely, so it can be used to extract the energy of a narrow band signal, such as the specific frequency components and high frequency components.

$$
E=\int_{\substack{t_{1} \leq t \leq t_{2} \\ f_{1} \leq f \leq f_{2}}} \operatorname{SST}^{2}(x(t))
$$

For the shunt capacitor with small damping reactance at the inverter station in the Tianzhong AC-DC hybrid system, its resonant frequency $f_{r}$ is:

$$
f_{\mathrm{r}}=\frac{1}{2 \pi \sqrt{L C}}=2048.5 \mathrm{~Hz}
$$

A flowchart of the boundary protection algorithm is shown in Fig. 10.

\section{Simulation and results analysis}

Based on the Tian-Zhong (in China) $\pm 800 \mathrm{kV} / 8000$ MW HVDC system, a typical AC-DC hybrid simulation model is established as shown in Fig. 1. Twenty-fourpulse converter valves are installed in the converter station. The lengths of the HVDC transmission line and protected AC transmission line, both modelled using frequency dependent models, are $2190 \mathrm{~km}$ and $62.5 \mathrm{~km}$, respectively. The other AC transmission lines are $21.27 \mathrm{~km}$ and $16.87 \mathrm{~km}$ long. The transmission line structures are shown in Fig. 11 (a) and (b) for AC and DC, respectively. As for control strategies, the rectifier station adopts the conventional constant current control strategy, and the inverter station adopts conventional constant current control and constant extinction angle control strategies.

The sampling rate is $400 \mathrm{kHz}$ and the total data window $\Delta t=1.28 \mathrm{~ms}$. The specific frequencies take the two center frequencies closest to $f_{r}$, i.e., $f_{O}=2040.2 \mathrm{~Hz}$ and $f_{1}=2087.3 \mathrm{~Hz}$. The high frequency components are taken from 30 to $35 \mathrm{kHz}$, i.e., $f_{l}=30 \mathrm{kHz}$ and $f_{h}=35 \mathrm{kHz}$. The protected line is $\mathrm{MN}$ and the boundary protection

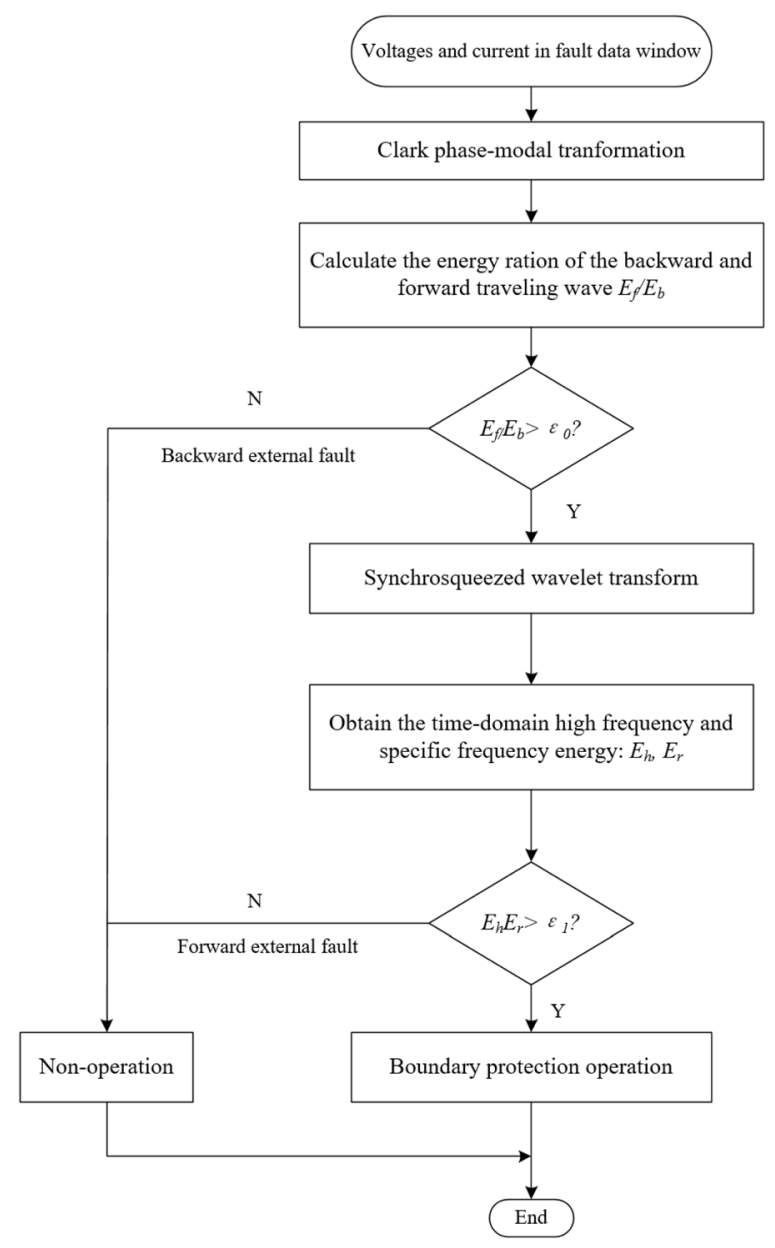

Fig. 10 Flowchart of boundary protection algorithm 


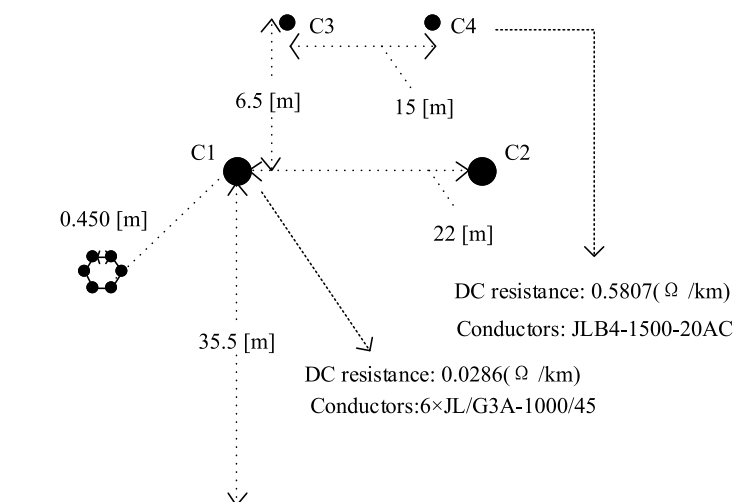

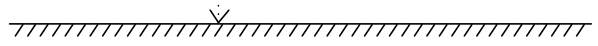

(a)

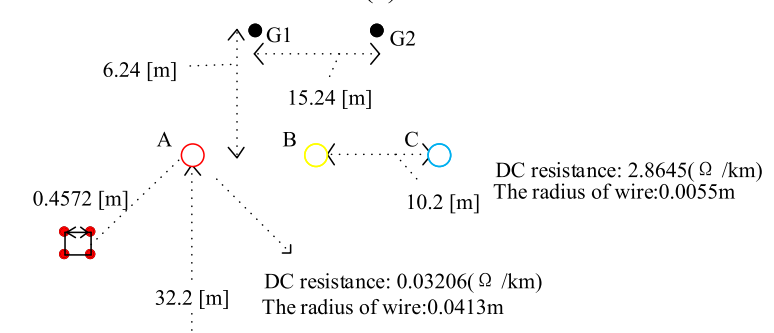

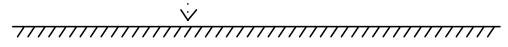

(b)

Fig. $11 \mathrm{AC}$ and HVDC transmission line structures

is arranged at the $\mathrm{N}$ end. For MMF, a flat structure element is employed and the size is 3 . The data window of the direction element $\Delta t$ dir $=0.15 \mathrm{~ms}\left(N_{d i r}=60\right)$ and the threshold $\varepsilon_{0}=2$. The setting method for boundary protection of an adaptive fault type is adopted, and the threshold $\varepsilon_{1}$ is respectively determined in terms of fault
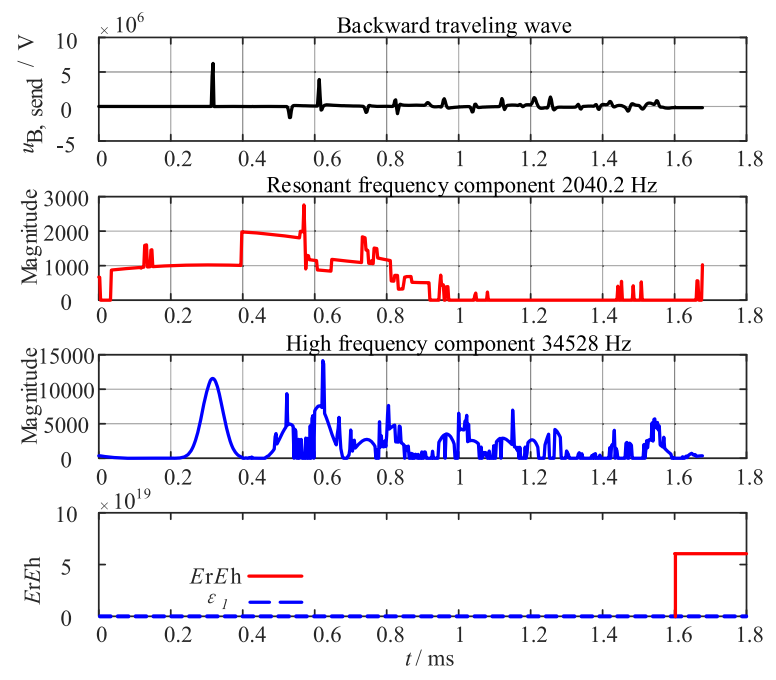

Fig. 12 Protection response for a typical internal fault
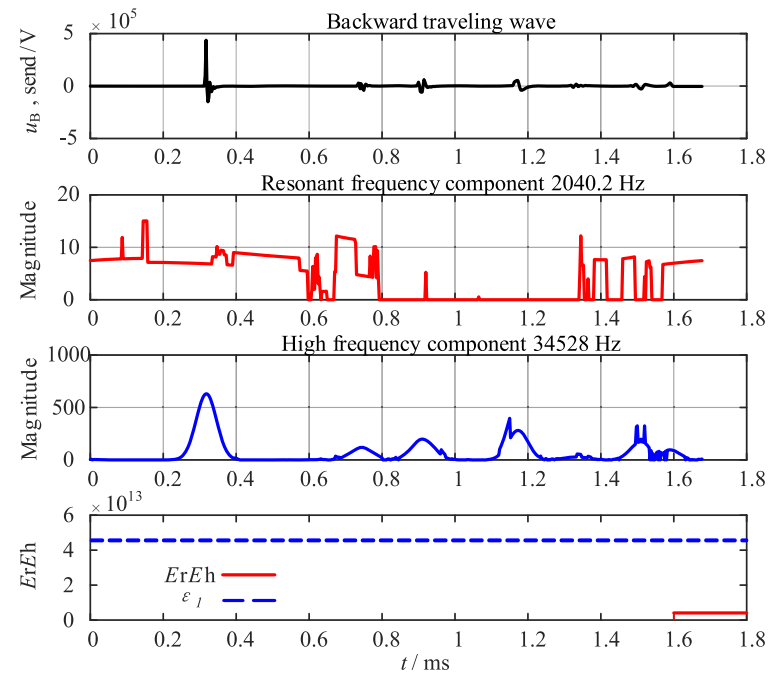

Fig. 13 Protection response for a typical external fault

types: $\varepsilon_{\phi \mathrm{g}}=4.56 \mathrm{e} 13, \varepsilon_{\phi \phi \mathrm{g}}=5.65 \mathrm{e} 15, \varepsilon_{\phi \phi}=7.32 \mathrm{e} 15$, and $\varepsilon_{\mathrm{ABC}}=1.25 \mathrm{e} 16$.

For an internal metallic phase A grounding fault at the middle of line $\mathrm{MN}$, which is $32.5 \mathrm{~km}$ from busbar $\mathrm{N}$ with the inception angle of $90^{\circ}$, as shown in Fig. 12, the fault occurs at $0.32 \mathrm{~ms}$ and the total fault data is $1.28 \mathrm{~ms}$ $(0.32-1.6 \mathrm{~ms})$. The fault direction is identified as a forward fault because of the energy ratio of the forward and backward traveling wave being $E_{\mathrm{f}} / E_{\mathrm{b}}=0.401034$. At $1.6 \mathrm{~ms}$, it calculates $E_{h} E_{r}=6.05 \mathrm{e} 19$, which is larger than the threshold $\varepsilon_{\phi \mathrm{g}}$. Thus, at only $1.28 \mathrm{~ms}$ after the fault occurrence, boundary protection operates.

For a forward external metallic phase A grounding fault at the middle of another line MQ at $0.32 \mathrm{~ms}$, which is $15 \mathrm{~km}$ from busbar $\mathrm{M}$ with phase $\mathrm{A}$ inception angle of
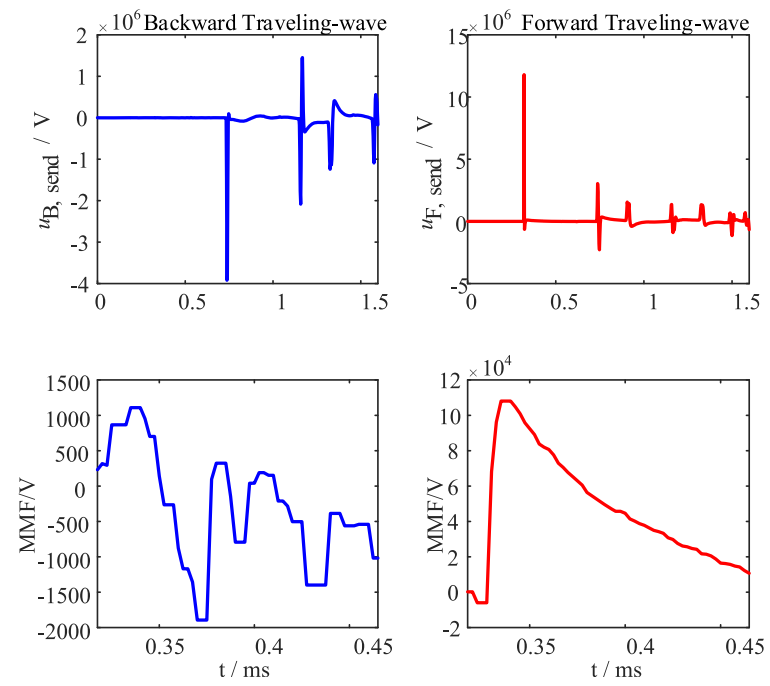

Fig. 14 Protection response for a typical backward external fault 
Table 3 High frequency and specific frequency energy of backward traveling wave at different fault locations $\left(R=300 \Omega, \theta=90^{\circ}\right.$, $\left.A B g\right)$

\begin{tabular}{lllll}
\hline Fault location & $\boldsymbol{E}_{\boldsymbol{h}}$ & $\boldsymbol{E}_{\boldsymbol{r}}$ & $\boldsymbol{E}_{\boldsymbol{h}} \boldsymbol{E}_{\boldsymbol{r}}$ & Discriminated result \\
\hline$I_{N f}=1 \mathrm{~km}$ & $1.5494 \mathrm{e}+10$ & $1.5841 \mathrm{e}+08$ & $2.4543 \mathrm{e}+18$ & Internal \\
$I_{N f}=31.25 \mathrm{~km}$ & $1.1377 \mathrm{e}+10$ & $2.0587 \mathrm{e}+08$ & $2.3421 \mathrm{e}+18$ & Internal \\
$I_{N f}=61.5 \mathrm{~km}$ & $1.6953 \mathrm{e}+10$ & $6.2233 \mathrm{e}+07$ & $1.0550 \mathrm{e}+18$ & Internal \\
\hline
\end{tabular}

$90^{\circ}$, the results are shown in Fig. 13. The fault direction is identified as a forward fault because of the energy ratio of the backward and forward traveling waves $E_{\mathrm{f}} / E_{\mathrm{b}}=$ 0.534551 . At $1.6 \mathrm{~ms}$, it calculates $E_{h} E_{r}=4.12 \mathrm{e} 12$, which is less than the threshold $\varepsilon_{\phi \mathrm{g}}$. Thus, boundary protection does not operate.

For a backward external phase A grounding fault at busbar $N$ at $0.32 \mathrm{~ms}$, with the fault resistance of $5 \Omega$ and the phase A inception angle of $90^{\circ}$, the results are shown in Fig. 14. As can be seen, the data window time for calculating $E_{f} / E_{b}$ is $0.32-0.47 \mathrm{~ms}$, and only $0.32 \mathrm{~ms}$ after the fault occurrence. It calculates $E_{f} / E_{b}=1.6 \mathrm{e} 6$, which is larger than the threshold $\varepsilon_{O}$ up to six orders of magnitude. Thus, the fault is reliably determined to be backward, and boundary protection does not operate.

The boundary protection is further tested under the following different fault conditions (f indicates the fault point) and Tables 3,4 and 5 summarize the simulation results of the boundary protection:

- Three fault positions in line MN: $l_{N f}=1 \mathrm{~km}, 31.25$ $\mathrm{km}$ and $61.5 \mathrm{~km}$;

- Two fault resistances (R): $0 \Omega$ and $300 \Omega$.

- Three phase-A inception angles $(\theta): 5^{\circ}, 45^{\circ}$ and $90^{\circ}$.

- Four fault types: A-G, AB-G, AB and ABC.

The simulation results of boundary protection with different fault locations, different fault resistances and different inception angles are given in Tables 3, 4 and 5. The smaller the initial fault angle is, the smaller the $E_{h} E_{r}$ value is, and the sensitivity of boundary element discrimination will decrease. When the fault with high resistance and small inception angle occurs at the end of the line, the boundary element will refuse to operate.

The extensive simulations in Tables 3, 4 and 5 show that most forward internal and external faults can be distinguished correctly within $1.28 \mathrm{~ms}$ after a fault occurrence. Only for single-phase grounding faults at the end of the protected line with high fault resistance or small fault inception angle, the sensitivity of boundary protection is reduced and boundary protection may fail to operate (such as the phase A grounding fault with $l_{N f}=62.5 \mathrm{~km}$ and the inception angle of $5^{\circ}$ in Table 5). It also indicates that it is difficult to reliably protect the whole line by only utilizing the non-unit transient fault information. The simulation results prove that the novel protection principle is immune to commutation failure, fault resistance and fault type.

\section{Conclusions}

This paper comprehensively analyzes the inherent frequency characteristics of an inverter station boundary. Both the AC boundary and DC boundary have significant attenuation on high frequency components and specific frequency components. The AC filters, shunt capacitors with small damping reactance and DC filters have attenuation on specific frequency components dependent on the structure of the filters, whereas shunt capacitors without small damping reactance and SR have significant attenuation on high frequency components.

Based on the boundary characteristics, a novel boundary protection principle using a backward traveling wave is proposed for an ACTL connected to an inverter station. As this protection scheme does not need a

Table 4 High frequency and specific frequency energy of backward traveling wave with different fault resistances $\left(\theta=90^{\circ}\right)$

\begin{tabular}{|c|c|c|c|c|c|c|}
\hline \multicolumn{3}{|l|}{ Fault condition } & \multirow[t]{2}{*}{$E_{h}$} & \multirow[t]{2}{*}{$E_{r}$} & \multirow[t]{2}{*}{$E_{h} E_{r}$} & \multirow{2}{*}{$\begin{array}{l}\text { Discriminated } \\
\text { result }\end{array}$} \\
\hline Fault location & $R / \Omega$ & Fault type & & & & \\
\hline \multirow[t]{8}{*}{$I_{N f}=31.25 \mathrm{~km}$} & 0 & $\mathrm{Ag}$ & $7.5379 e+10$ & $8.0295 e+08$ & $6.0526 e+19$ & Internal \\
\hline & & $A B$ & $9.6894 e+10$ & $1.1590 \mathrm{e}+09$ & $1.1230 \mathrm{e}+20$ & Internal \\
\hline & & $\mathrm{ABg}$ & $1.0511 \mathrm{e}+11$ & $1.0678 \mathrm{e}+09$ & $4.2761 e+19$ & Internal \\
\hline & & $A B C$ & $1.6995 \mathrm{e}+11$ & $2.2635 e+09$ & $1.1224 \mathrm{e}+20$ & Internal \\
\hline & 300 & $\mathrm{Ag}$ & $1.0334 \mathrm{e}+10$ & $2.4429 e+08$ & $2.5243 e+18$ & Internal \\
\hline & & $A B$ & $1.7593 e+10$ & $4.8874 \mathrm{e}+08$ & $8.5986 e+18$ & Internal \\
\hline & & $\mathrm{ABg}$ & $1.1377 \mathrm{e}+10$ & $2.0587 e+08$ & $2.3421 \mathrm{e}+18$ & Internal \\
\hline & & $A B C$ & $4.3934 \mathrm{e}+10$ & $6.7390 e+08$ & $2.9607 e+19$ & Internal \\
\hline
\end{tabular}


Table 5 Simulation results of boundary protection with different fault inception angles (Ag)

\begin{tabular}{|c|c|c|c|c|c|c|}
\hline \multicolumn{3}{|l|}{ Fault condition } & \multirow[t]{2}{*}{$E_{h}$} & \multirow[t]{2}{*}{$E_{r}$} & \multirow[t]{2}{*}{$E_{h} E_{r}$} & \multirow{2}{*}{$\begin{array}{l}\text { Discriminated } \\
\text { result }\end{array}$} \\
\hline Fault location & $\theta$ & $R / \Omega$ & & & & \\
\hline \multirow[t]{6}{*}{$I_{\mathrm{Nf}}=1 \mathrm{~km}$} & $90^{\circ}$ & 0 & $9.6291 e+10$ & $9.3291 e+09$ & $8.9832 e+20$ & Internal \\
\hline & & 300 & $8.9699 e+09$ & $1.2171 \mathrm{e}+08$ & $1.0918 \mathrm{e}+18$ & Internal \\
\hline & $45^{\circ}$ & 0 & $4.9697 \mathrm{e}+10$ & $8.6482 e+09$ & $4.2979 e+20$ & Internal \\
\hline & & 300 & $4.6459 e+09$ & $6.2445 e+07$ & $2.9011 \mathrm{e}+17$ & Internal \\
\hline & $5^{\circ}$ & 0 & $6.5556 \mathrm{e}+08$ & $1.6960 \mathrm{e}+08$ & $1.1118 \mathrm{e}+17$ & Internal \\
\hline & & 300 & $6.0405 e+07$ & $2.5661 e+06$ & $1.5500 \mathrm{e}+14$ & Internal \\
\hline \multirow[t]{6}{*}{$I_{\mathrm{Nf}}=31.25 \mathrm{~km}$} & $90^{\circ}$ & 0 & $7.5379 \mathrm{e}+10$ & $8.0295 e+08$ & $6.0526 e+19$ & Internal \\
\hline & & 300 & $1.0334 \mathrm{e}+10$ & $2.4429 e+08$ & $2.5243 e+18$ & Internal \\
\hline & $45^{\circ}$ & 0 & $3.6900 \mathrm{e}+10$ & $4.1460 \mathrm{e}+08$ & $1.5299 e+19$ & Internal \\
\hline & & 300 & $5.1037 \mathrm{e}+09$ & $1.2447 \mathrm{e}+08$ & $6.3526 e+17$ & Internal \\
\hline & $5^{\circ}$ & 0 & $5.2071 \mathrm{e}+08$ & $8.5259 e+06$ & $4.4395 e+15$ & Internal \\
\hline & & 300 & $7.1285 e+07$ & $2.3735 e+06$ & $1.6919 e+14$ & Internal \\
\hline \multirow[t]{6}{*}{$I_{\mathrm{Nf}}=61.5 \mathrm{~km}$} & $90^{\circ}$ & 0 & $3.8895 e+10$ & $4.9823 e+08$ & $1.9378 \mathrm{e}+19$ & Internal \\
\hline & & 300 & $1.0006 \mathrm{e}+10$ & $8.0355 e+05$ & $8.0407 e+15$ & Internal \\
\hline & $45^{\circ}$ & 0 & $1.9488 \mathrm{e}+10$ & $3.0777 \mathrm{e}+08$ & $5.9977 e+18$ & Internal \\
\hline & & 300 & $4.9731 \mathrm{e}+09$ & $3.2226 \mathrm{e}+05$ & $1.6026 \mathrm{e}+15$ & Internal \\
\hline & $5^{\circ}$ & 0 & $3.1253 e+08$ & $5.1877 e+07$ & $1.6213 e+16$ & Internal \\
\hline & & 300 & $8.0297 e+07$ & $4.5757 e+04$ & $3.6742 \mathrm{e}+12$ & external \\
\hline
\end{tabular}

communication channel to receive information from the remote end, it can identify a fault in under $3 \mathrm{~ms}$. Therefore, the consequent commutation failure will not affect its correct detection of the fault. Simulation results show that the boundary protection operates stably under various fault conditions.

However, it is difficult to reliably protect the whole line under some heavy fault conditions and further studies and explorations are needed to improve this scheme.

\section{Acknowledgements}

Not applicable.

\section{About the authors}

Z. Liu (1995-), male, received the B.Sc. Degrees in electrical engineering from Shandong University, is currently working toward the Ph.D. degree at Shandong University. Major in the power system fault analysis and protection, and HVDC protection. E-mail:zliu.sdu@gmail.com.

H. L. Gao (1963-), received the B.S. and M.S. degrees in electrical power engineering from Shandong University of Technology, Jinan, China, in 1983 and 1988, respectively, and the Ph.D. degree in electrical power system and automation from Tianjin University, Tianjin, China, in 1997. From 2004 to 2005, he worked as senior visiting scholar with the School of Electrical and Electronic Engineering, Queen's University Belfast, U.K. He is currently a Professor at the School of Electrical Engineering, Shandong University, China. He has authored or coauthored more than 170 technical papers. His research interests include power system protection, fault location, smart substation and distributed generation. E-mail: houleig@sdu.edu.cn.

S. B. Luo (1985-), male, PHD, Major in power system fault analysis and protection. E-mail: starbayer@163.com.

L. Zhao, male, a native of Jingzhou, Hubei, works at the Electric Power Research Institute of State Grid Shanghai Electric Power Company, specializing in UHVDC software control protection simulation technology, and new energy power electronics hardware simulation technology in the loop. E-mail: zhaole@sh.sgcc.com.cn.

Y. Y. Feng, male, born in Shanghai, senior engineer, graduated from Shanghai Jiaotong University. His research fields include power system, large power grid safety and stability simulation analysis, UHVAC and DC hybrid simulation analysis. E-mail: fengyy@sh.sgcc.com.cn.

\section{Authors' contributions}

ZL established the LCC-HVDC simulation model, conducted the fault simulation, tested the boundary protection algorithm and drafted major part of the manuscript. HG proposed the idea to apply boundary protection in AC line connected to inverter station and drafted the structure of the manuscript. SL analyzed the frequency characteristics of AC transmission line boundary connected to LCC-HVDC inverter station, figured out the specific frequency components, and drafted some of the manuscript. LZ and YF shared some onsite commutation failure recording data. The authors read and approved the submitted manuscript. The content of the manuscript has not been published.

\section{Funding}

This work was supported by the National Key R\&D Program of China (2016YFB0900603) and the Technology Projects of State Grid Corporation of China (52094017000 W).

\section{Availability of data and materials}

Not applicable.

\section{Competing interests}

The authors declare that they have no competing interests.

\section{Author details}

${ }^{1}$ Key Laboratory of Power System Intelligent Dispatch and Control of Ministry of Education, Shandong University, No. 17923, Jingshi Road, Jinan 250061, China. ${ }^{2}$ Electric Power Research Institute of State Grid Shanghai Electric Power Company, No. 171, Handan Road, Shanghai, China. 
Received: 11 April 2020 Accepted: 28 October 2020

Published online: 27 November 2020

\section{References}

1. Luo, S. B., et al. (2018). Non-unit transient based boundary protection for UHV transmission lines. International Journal of Electrical Power \& Energy Systems, 102, 349-363.

2. Zheng, J., et al. (2018). A novel differential protection scheme for HVDC transmission lines. International Journal of Electrical Power \& Energy Systems, 94, 171-178,

3. Chen, G., et al. (2015). Review of high voltage direct current cables. CSEE Journal of Power and Energy Systems, 1(2), 9-21.

4. Zhang, Y., Tai, N., \& Xu, B. (2012). Fault analysis and traveling-wave protection scheme for bipolar HVDC lines. IEEE Transactions on Power Delivery, 27(3), 1583-1591.

5. Zou, G., et al. (2018). A fast protection scheme for VSC based multiterminal DC grid. International Journal of Electrical Power \& Energy Systems, 98, 307-314.

6. Lin, et al. (2018). A review on protection of DC microgrids. Journal of Modern Power Systems and Clean Energy, 6(06), 1113-1127.

7. Shao-Feng, H., et al. (2014). Effect of commutation failure on distance protection and the countermeasures. Power System Protection and Control, 42(20):123-128.

8. Meah, K., \& Ula (2010). A new simplified adaptive control scheme for multiterminal HVDC transmission systems. International Journal of Electrical Power \& Energy Systems, 32(4), 243-253.

9. Wei, Z., et al. (2014). Direct-current predictive control strategy for inhibiting commutation failure in HVDC converter. IEEE Transactions on Power Apparatus and Systems, 29(5), 2409-2417.

10. Wang, D., et al. (2017). Travelling wave pilot protection for LCC-HVDC transmission lines based on electronic transformers' differential output characteristic. International Journal of Electrical Power \& Energy Systems, 93, 283-290.

11. Qiang, L., et al. (2008). Influence of HVDC commutation failure on directional comparison pilot protection of AC system. In International conference on electric utility deregulation \& Restructuring \& Power Technologies IEEE.

12. Huanhuan, et al. (2018). Assessment of commutation failure in HVDC systems considering spatial-temporal discreteness of AC system faults. Journal of Modern Power Systems and Clean Energy, 6(05), 1055-1065.

13. Junlei, L., et al. (2013). Mechanism analysis of HVDC commutation failure influence on $A C$ power network relay protection. Proceedings of the CSEE, 33(19), 111-118.

14. Shen, H., Huang, S., \& Fei, B. (2015). Transient characteristic of HVDC system during commutation failure, its effect on differential protection and countermeasures. Electric Power Automation Equipment, 35(4), 109114 and 120

15. Dong, W., et al. (2018). Adaptability analysis of directional pilot protection for AC transmission lines connected to LCC-HVDC inverter station. Power System Protection and Control, 46(18):33-40.

16. Zhang, J., et al. (2013). Performance analysis of distance protection based on industrial frequency variation applied to AC-DC hybrid power grid. Automation of Electric Power Systems, 37(4), 98-103.

17. Qiu, Y. T., et al. (2012). Research on applicability of directional element based on the power-frequency variation in AC-DC hybrid system. Power System Protection \& Control, 40(13):115-120.

18. Hongming, S., Shaofeng, H., \& Bin, F. (2015). Effect analysis of AC/DC interconnected network on distance protection performance and countermeasures. Automation of Electric Power Systems, 39(11), 58-63 and 82.

19. De-Feng, L. U., Wei-Min, M., \& Wei-Xiong, X. (2006). Discussion on incorrect action of protection caused by commutation failure in DC Converter Station and its countermeasures. Electrical Equipment, (01):54-56.

20. Zhiyao, L., et al. (2006). Analysis of relay protection Misoperation caused by DC commutation failure. Automation of Electric Power Systems, 30(19), 104-107.

21. Chamia, M., \& Liberman, S. (1978). Ultra high speed relay for EHV/UHV transmission lines development, design and application. IEEE Transactions on Power Apparatus and Systems, PAS-97(6), 2104-2116.
22. Johns, A. T. (1980). New ultra-high-speed directional comparison technique for the protection of e.h.v. transmission lines. IEE Proceedings C - Generation, Transmission and Distribution, 127(4), 228-239.

23. Crossley, P. A., \& Mclaren, P. G. (2007). Distance protection based on travelling waves. Power Engineering Review IEEE, PAS-102(9), 2971-2983.

24. Dong, X., Ge, Y., \& He, J. (2005). Surge impedance relay. IEEE Transactions on Power Delivery, 20(2), 1247-1256.

25. Zou, G. B., \& Gao, H. L. (2013). Fast pilot protection method based on waveform integral of traveling wave. International Journal of Electrical Power \& Energy Systems, 50, 1-8.

26. Song, G., et al. (2015). A new whole-line quick-action protection principle for HVDC transmission lines using one-end current. IEEE Transactions on Power Delivery, 30(2), 599-607.

27. Li, B., He, J., Li, Y. et al. (2019). A review of the protection for the multiterminal VSC-HVDC grid. Protection and Control of Modern Power Systems, 4(1), 21. https://doi.org/10.1186/s41601-019-0136-2.

28. Johns, A. T., Aggarwal, R. K., \& Bo, Z. Q. (1994). Non-unit protection technique for EHV transmission systems based on fault-generated noise. Part 1: Signal measurement. Generation Transmission \& Distribution lee Proceedings, 141(2), 133-140.

29. Aggarwal, R. K., \& Johns, A. T. (1994). Non-unit protection technique for EHV transmission systems based on fault-generated noise. Part 2: Signal processing. IEE Proceedings Part C, 141(2), 141-147.

30. Bo, Z. Q. (1998). A new non-communication protection technique for transmission lines. IEEE Transactions on Power Delivery, 13(4), 1073-1078.

31. Duan, J. D., Zhang, B. H. Li, P., et al. (2007). Principle and algorithm of nonunit transient-based protection for EHV transmission lines. Zhongguo Dianji Gongcheng Xuebao/Proceedings of the Chinese Society of Electrical Engineering, 27(7), 45-51.

32. Liu, X., Osman, A. H., \& Malik, O. P. (2009). Hybrid traveling wave/boundary protection for bipolar HCDC line. IEEE Transactions on Power Delivery, 24(2), $569-578$.

33. Liu, X. Osman, A. H., \& Malik, O. P. (2011). Real-time implementation of a hybrid protection scheme for bipolar HVDC line using FPGA. IEEE Transactions on Power Delivery, 26(1), 101-108.

34. Kong, F., Hao, Z., Zhang, S., et al. (2014). Development of a novel protection device for bipolar HVDC transmission lines. IEEE Transactions on Power Delivery, 29(5), 2270-2278.

35. Kong, F., Hao, Z., \& Zhang, B. (2016). A novel traveling-wave-based Main protection scheme for $+/-800$ kV UHVDC bipolar transmission lines. IEEE Transactions on Power Delivery, 31(5), 1.

36. Xiao, H., Li, Y., Liu, R., et al. (2017). Single-end time-domain transient electrical signals based protection principle and its efficient setting calculation method for LCC-HVDC lines. IET Generation Transmission and Distribution, 11(5), 1233-1242.

37. Megahed, A. I., Monemmoussa, A., \& Bayoumy, A. E. (2006). Usage of wavelet transform in the protection of series-compensated transmission lines. IEEF Transactions on Power Delivery, 21(3), 1213-1221.

38. Zhang, N., \& Kezunovic, M. (2007). Transmission line boundary protection using wavelet transform and neural network. IEEE Transactions on Power Delivery, 22, 859-869

39. Khodadadi, M., \& Shahrtash, S. (2013). A new non-communication based protection scheme for three-terminal; transmission lines employing mathematical morphology-based filters. IEEE Transactions on Power Delivery, 28(2), 1247.

40. Musa, M.H.H., He, Z., Fu, L. et al. (2018). A cumulative standard deviation sum based method for high resistance fault identification and classification in power transmission lines. Protection and Control of Modern Power Systems, 3(1), 30. https://doi.org/10.1186/s41601-018-01024.

41. Jinyu, L., et al. (2018). Modeling and fault simulation of the $\pm 800 \mathrm{kV}$ UHVDC system. Foreign Electronic Measurement Technology, 37(05):1-7.

42. $\mathrm{Yu}, \mathrm{T}$. (2015). Analysis and application of DC transmission control protection system, China: Beijing, (pp. 108-110). 